\title{
CEREBELLAR INFARCTS AND COGNITIVE DEFICITS
}

Twenty-four young adults with infratentorial infarcts were evaluated by MRI and angiography and assessed by neuropsychological tests, acutely and at 4 and 12 months after the stroke, in a prospective study at the University of Umea, Sweden. Twenty two had a favorable outcome, but most performed worse than controls on tasks of attention, visuospatial skills, working memory, and cognitive flexibility. IQ scores were related to size of infarct, and early post-stroke, block design task performance predicted maximal work performance at 12 months. Standard tests of intelligence and episodic memory were unchanged, and the neurologic examination was normal. However, only $57 \%$ of patients were at work full-time 1 year after the stroke. Headache, fatigue, anxiety, and memory disturbance contributed to the disability. (Malm J, Kristensen B, Karlsson T et al. Cognitive impairment in young adults with infratentorial infarcts. Neurology Aug 1998;51:433-440). (Reprints: Dr Jan Malm, Department of Clinical Neuroscience, University Hospital, S-901 85 Umea, Sweden).

COMMENT. Cerebellar damage from infarcts in young adults impairs attention, working memory, and visuospatial skills, while sparing, intelligence and episodic memory. Working memory involves short-term, temporary, and recalled experiences used to support other mental functions. Whereas digit and word span tasks requiring passive repetition are unaffected by cerebellar damage, a more demanding and complex listening span task, requiring repetition of words while simultaneously trying to analyze sentences, is significantly impaired.

Hypoplasia of the cerebellar vermis following cranial irradiation for acute leukemia has been correlated with cognitive deficits involving visuospatial coordination and memory while sparing language and verbal processing (see Progress in Pediatric Neurology III, 1997; pp423-4). The cerebellum and/or its connections with fronto-striate regions have important cognitive functions.

\section{COGNITIVE AND AUTISTIC EPILEPTIFORM REGRESSION}

Seven children, ages 3 to 8 years, presenting with autism or autistic regression and seizures had occipital spikes, characteristic of benign epilepsies, in a report from New York University Medical Center. Clinical findings included borderline or mental retardation, delayed or regressing language development, autistic and hyperactive behavior, and chronic motor tics. Seizures consisted of absence or myoclonic patterns, usually resistant to antiepileptic drugs. A causal relation between occipital spikes and cognitive and language regression was suggested, reflecting extension of epileptiform activity into temporal and posterior-parietal areas. (Nass R, Gross A, Devinsky O. Autism and autistic epileptiform refression with occipital spikes. Dev Med Child Neurology July 1998;40:453-458). (Respond: Dr Ruth Nass, Department of Neurology, New York University Medical Center, 400 East 34th St, NYC, NY 10016).

COMMENT. The relation between EEG epileptiform abnormalities and cognitive, language, and behavioral disorders is controversial. The correlation between the seizure, behavioral, and language disorders in children with LandauKleffner syndrome and autistic epileptiform regression is debatable. A temporal relation between treatment with antiepileptic drugs and clinical improvement is sometimes supportive, but responses are often unsustained. This report is the first to relate occipital spikes or spike-wave epileptiform EEGs to cognitive, language, and autistic regression. See Progress in Pediatric Neurology II, 1994;pp220-226. 